\title{
Nuevas formas farmacéuticas para el tratamiento de enfermedades alérgicas
}

\section{New pharmaceutical dosage forms for allergy treatment}

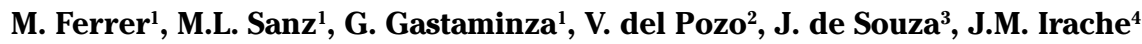

\section{RESUMEN}

La inmunoterapia para el tratamiento de enfermedades alérgicas implica ciertas desventajas, que pueden ser reducidas si se emplean adyuvantes adecuados, que sean capaces de amplificar la respuesta inmune con un efecto alergénico mínimo. En ese contexto, las formas farmacéuticas más prometedoras para aumentar la eficacia y seguridad de la inmunoterapia, parecen ser las micro y nanopartículas, de polímeros biodegradables y liposomas. En esta revisión describimos estudios previos de nuestro grupo en los que empleamos como adyuvante nanopartículas Gantrez ${ }^{\circledR}$ AN y demostramos su capacidad de estimular el sistema inmune. Empleamos dos tipos de nanopartículas, con y sin lipopolisacárido de Brucella ovis como inmunomodulador en un modelo de ratón alérgico a $L$. perenne. Encontramos que los ratones sensiblizados a Phleum cuando recibían inmunoterapia con nanopartículas Lolium-Gantrez ${ }^{\circledR}$ estaban protegidos de la anafilaxia inducida por el alérgeno tanto en las tasas de mortalidad como en los niveles de MCP-1. Probamos asimismo estas formulaciones por vía oral en un modelo animal sensibilizado a ovoalbúmina y comprobamos que les protegía también del shock anafiláctico.

Palabras clave. Inmunoterapia. Alergia. Nanopartículas. Micropartículas.

\begin{abstract}
Specific immunotherapy involves certain drawbacks which could be minimized by the use of appropriate adjuvants, capable of amplifying the right immune response with minimal side effects. In this context, we review different types of immunotherapy vehicles and coadyuvants. We describe previous studies by our group in which we demonstrated the adjuvant capacity of Gantrez® AN nanoparticles, which can effectively enhance the immune response. We employed two types of nanoparticles (with and without LPS of Brucella ovis as immunomodulator) within capsulated ovoalbumin and Lollium perenne extract, tested on a model of mice sensitized to this allergenic mixture. In the challenge experiment involving the sensitized mice, differences in the mortality rate and in the MCP-1 levels were found between the treated groups and the control. Under the experimental conditions of this model of mice pre-sensitized to $L$. perenne, Gantrez ${ }^{\circledR} A N$ nanoparticles appeared to be a good strategy for immunotherapy. We finally tested these carriers administered by the oral route and found that they were able to protect a model of mice sensitized to ovalbumin from anaphylactic shock.
\end{abstract}

Key words. Immunotherapy. Allergy. Nanoparticles. Microparticles.
1. Departamento de Alergología, Clínica Universidad de Navarra, Facultad de Medicina, Universidad de Navarra, Pamplona

2. Departamento de Inmunología, Fundación Jiménez Díaz, Madrid

3. Departamento de Microbiología, Facultad de Medicina, Universidad de Navarra, Pamplona

4. Departamento de Farmacia y Tecnología Farmacéutica, Facultad de Farmacia, Universidad de Navarra, Pamplona

Recepción: 25 de mayo de 2011

Aceptación provisional: 23 de junio de 2011

Aceptación definitiva: 25 de octubre de 2011

\section{Correspondencia: \\ Marta Ferrer Puga \\ Departamento de Alergología \\ Clínica Universidad de Navarra \\ Pio XII, 36 \\ 31008-Pamplona \\ Email: mferrerp@unav.es}

Esta investigación ha estado financiada por el proyecto de investigación del Ministerio de Ciencia y Tecnología (SAF 2001-0690-C03-01) y por el proyecto del Gobierno de Navarra (28/2007). Está financiada por el Instituto de Salud Carlos III (proyecto: PS09/01083) y la Fundación Ramón Areces. Juliana de Souza está asimismo financiada por una beca de la Asociación de Amigos de la Universidad de Navarra. 


\section{INTRODUCCIÓN}

Las enfermedades alérgicas son una patología emergente. Existe un $20 \%$ de prevalencia de sensibilización alérgica ${ }^{1,2}$. En un reciente estudio realizado en nuestro país por la Sociedad Española de Alergología sobre los factores epidemiológicos, clínicos y socio-económicos de las enfermedades alérgicas, se ha calculado que un $7,4 \%^{3}$ y un $22 \%$ de pacientes que acuden por primera vez a un alergólogo lo hacen por alergia alimentaria y rinitis respectivamente . $^{4}$

El único tratamiento capaz de modificar la respuesta natural es la inmunoterapia.

La eficacia de la inmunoterapia ha sido demostrada científicamente en numerosos ensayos doble ciego controlados con placebo, y así lo demuestran los últimos meta análisis ${ }^{5,6}$.

\section{NUEVAS FORMAS FARMACÉUTICAS}

En este contexto, cada vez están tomando más importancia los adyuvantes ${ }^{7,8}$ que permitan la presentación del alérgeno a las células presentadoras de antígeno, desencadenando una respuesta innata que ponga en marcha una señal celular moduladora de la respuesta de hipersensibilidad que origine tolerancia y además sea segura. En muchos casos el mecanismo por el cual un adyuvante es capaz de aumentar o modular la inmunogenicidad de un alérgeno o de un antígeno se desconoce ${ }^{9}$. Por otra parte, a pesar de la larga lista de compuestos que han sido propuestos como adyuvantes, a fecha de hoy solamente las sales de aluminio y la emulsión MF59 están aceptados para su uso en humanos ${ }^{10}$. Las sales de aluminio (el adyuvante más utilizado hoy en día) son capaces de inducir razonables respuestas humorales (Th2), pero no producen repuestas celulares (Th1). La inmunidad Th1 es esencial para la protección frente a numerosos organismos infecciosos (parásitos intracelulares) y para limitar procesos alérgicos. Además, hay que tener en cuenta que la sales de aluminio presentan limitaciones en cuanto a su aplicación en vacunas basadas en péptidos o fracciones de $\mathrm{ADN}^{11,12}$.
En la actualidad, un gran número de compuestos diferentes están siendo estudiados como potenciales adyuvantes. Entre éstos se encuentran las nuevas formas farmacéuticas para vehiculización de moléculas biológicamente activas.

Hoy en día está establecido de forma fehaciente que los adyuvantes pueden aumentar la respuesta inmune específica de los antígenos/alergenos mediante dos mecanismos principales ${ }^{13,14}$ : (i) aumentando la captura del alergeno por las células presentadoras de antígeno (CPAs). Estos adyuvantes son directamente capturados y fagocitados por las CPAs o bien forman un depósito en el lugar de administración o de absorción que prolonga su exposición y, por lo tanto, su captura por las CPAs. En este grupo se incluyen las sales de aluminio, las emulsiones, los derivados de saponinas y las nuevas formas farmacéuticas (liposomas, nanopartículas y micropartí-

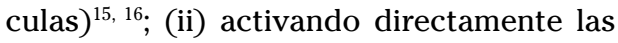
células del sistema inmune innato. A esta categoría pertenecen los estímulos inflamatorios como las citoquinas, los CD40L y los PAMPs (moléculas modelo asociadas a patógenos). Entre estos últimos se incluyen lipopolisacárido, mureína, flagelina, secuencias $\mathrm{CpGs}^{9}$.

Otra ventaja de algunas de las nuevas formas farmacéuticas, como ciertos tipos de nanopartículas, es que representan una buena estrategia para la administración segura y eficaz de alérgenos por vías a través de mucosas (incluida la vía oral). Además, durante los últimos años, ha aumentado el interés por las vías locales no inyectables buscando mejorar la seguridad y eficacia del tratamiento, y la aceptación del mismo por parte del paciente. La vía que ha demostrado eficacia y seguridad ha sido la vía sublingual para el tratamiento de rinitis y conjuntivitis ${ }^{17,18}$. Asimismo, la inmunoterapia sublingual frente a alimentos está en fase importante de desarrollo obteniéndose resultados prometedores ${ }^{19,20}$.

Otra vía interesante por su gran aceptación por parte de los pacientes es la vía oral. Para esta vía de administración la inducción de respuestas desensibilizantes o bloqueantes de reacciones alérgicas está 
asociado a (i) la protección del alérgeno frente a su degradación prematura y (ii) al desarrollo de interacciones bioadhesivas, entre la superficie de estos vehículos y la mucosa, que permitan incrementar el tiempo de residencia en esta zona ${ }^{21}$.

En ese contexto, las nuevas formas farmacéuticas más prometedoras como adyuvantes parecen ser las micro y nanopartículas $^{22-24}$ de polímeros biodegradables y ciertos tipos de liposomas ${ }^{25-28}$ que permiten diseñar una terapéutica más racional y mejor adaptada, al aumentar la eficacia y especificidad de la molécula biológicamente activa que incorporan. Todas estas formas farmacéuticas, también llamadas vehículos o transportadores, tienen capacidad de ser captadas eficientemente por las células presentadoras de antígeno ${ }^{29}$.

Desde un punto de vista general, se puede afirmar que estos vehículos o transportadores de moléculas biológicamente activas aportan las siguientes ventajas ${ }^{30}$ :

1. Proteger el material que incorporan frente a su inactivación química, enzimática o inmunológica.

2. Mejorar el transporte de la molécula biológicamente activa hasta lugares difíciles de alcanzar y de su penetración en la célula.

3. Aumentar la especificidad de acción por concentración selectiva, eficaz y regular del fármaco en el blanco celular y/o molecular. Así, con dosis más pequeñas, la actividad terapéutica obtenida es, al menos, idéntica y los efectos secundarios menores.

4. Disminuir la toxicidad para ciertos órganos mediante modificación de la distribución tisular de la molécula biológicamente activa transportada.

5. Prolongar el tiempo de residencia de la molécula activa en el organismo (interesante para aquellas moléculas con aclaramiento elevado y semivida biológica baja) y, control de su liberación. Todo ello implica disminuir la frecuencia de tomas e, indirectamente, aumentar la observancia del tratamiento por parte del paciente.
En la bibliografía se encuentran varios trabajos que demuestran la eficacia de la inmunoterapia con estos vehículos frente a alergia producida por polen de abedul ${ }^{31}$ u olivo ${ }^{32}$.

\section{LIPOSOMAS}

Los liposomas son vesículas lipídicas constituidas por una o múltiples bicapas concéntricas de fosfolípidos que se alternan con compartimentos acuosos. Estas bicapas están formadas generalmente por una mezcla de sustancias lipídicas, fundamentalmente fosfolípidos y colesterol. Los métodos de preparación de liposomas difieren unos de otros por el modo de dispersión de los lípidos en la fase acuosa ${ }^{33-35}$. Así, se pueden clasificar en: (i) métodos mecánicos de dispersión de fosfolípidos; (ii) métodos basados en la dispersión de una solución orgánica de fosfolípidos; y (iii) técnicas de dispersión de micelas mixtas, seguido por la eliminación de los fosfolípidos. La mayoría de estas técnicas implican operaciones complejas de dispersión de lípidos o emulsificación y posterior reducción y homogeneización del tamaño de las vesículas obtenidas.

Recientemente, la Unidad de Adyuvantes de la Universidad de Navarra ha puesto a punto una técnica original para la fabricación de liposomas por inyección coaxial turbulenta. Esta técnica patentada permite obtener fácilmente liposomas muy homogéneos de pequeño tamaño (Fig. 1), evitando operaciones suplementarias de homogeneización por extrusión mediante el uso de la prensa de French, aparatos de microfluidificación o "extruders". Igualmente, este nuevo procedimiento permite el trabajo en continuo y su transposición a escala industrial es relativamente fácil. Además, la inyección coaxial turbulenta ya se está utilizando para la fabricación de liposomas destinados a la administración sublingual de diferentes extractos alergénicos (gramíneas, ácaros, olea, etc.) (Alervaccine®, Inmunal SA). Es interesante reseñar que las vacunas con estos liposomas se han ensayado por vía parenteral en alergia respiratoria con buena respuesta ${ }^{36,37}$. 


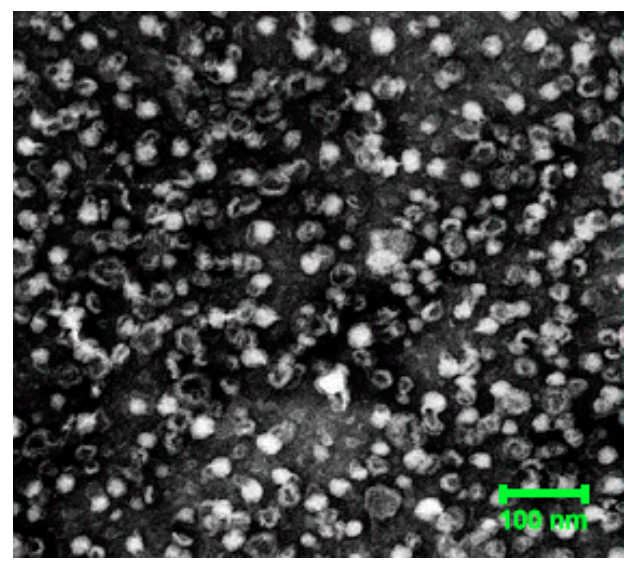

Figura 1. Liposomas obtenidos por inyección coaxial turbulenta.

\section{MICROPARTÍCULAS}

Las micropartículas poliméricas son formas farmacéuticas de tipo partícula sólida y de tamaño superior o igual al micrómetro y con estructura matricial porosa o vesicular $^{38}$. En general, las ventajas principales de las micropartículas son el ofrecer una protección al fármaco encapsulado frente a su eventual degradación en las condiciones de almacenamiento y/o biológicas y el permitir perfiles de liberación sostenida en el tiempo, sin necesidad de administraciones repetidas. Existen numerosas técnicas que permiten la fabricación de micropartículas, algunas de las cuales pueden utilizarse industrialmente. Entre estas cabe destacar la microencapsulación por lecho fluido, la microencapsulación por pulverización ("spray-drying") y la microencapsulación mediante fluidos en condiciones supercríticas.

Por otra parte, la Unidad de Adyuvantes de la Universidad de Navarra ha desarrollado y patentado dos técnicas, aplicables en el ámbito industrial, para la fabricación de micropartículas con distintas propiedades. Así, la inyección coaxial turbulenta permite la fabricación en continuo de microesferas de distintos poliésteres con un tamaño muy homogéneo y comprendido entre 2-5 $\mu \mathrm{m}$. Por otra parte, ha desarrollado también una técnica para la fabricación de micro- partículas capaces de encapsular moléculas hidrofílicas. La fabricación tiene lugar en un aparato denominado "TROMS" (Total Recirculation One-Machine System o Sistema Monobloque de Recirculación Completa) basado en la inyección turbulenta de dos fases para formar una doble emulsión (Fig. 2) y que permite obtener micropartículas de un tamaño prefijado (entre 1 y 100 $\mu \mathrm{m})$ y con una distribución muy uniforme. Una de las principales ventajas de este procedimiento radica en no exponer el material a encapsular a fuerzas de cizallamiento que pudieran inactivarlo, como puede ser el caso cuando se encapsulan proteínas o plásmidos por otras técnicas.

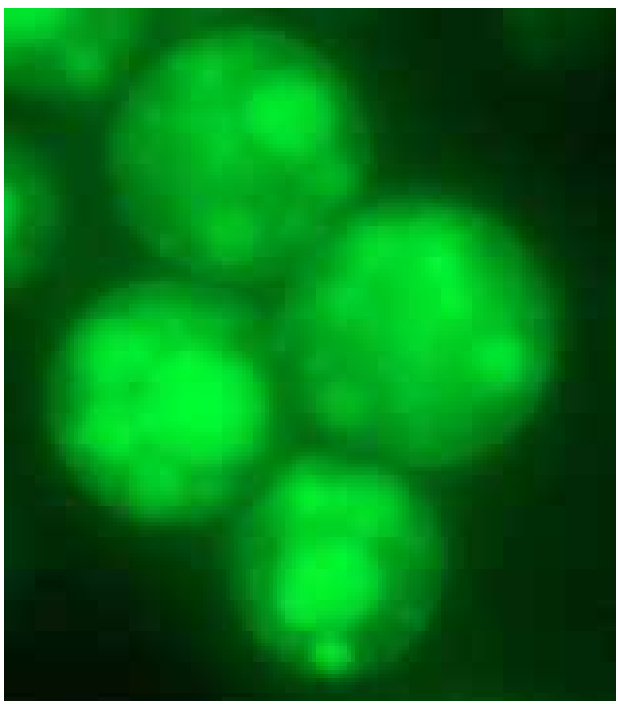

Figura 2. Emulsión múltiple de tipo A/O/A (agua en aceite en agua).

\section{NANOPARTÍCULAS}

Las nanopartículas (nanoesferas y nanocápsulas) son sistemas coloidales poliméricos, de tipo esférico y con un diámetro inferior a $1 \mu \mathrm{m}$. Administradas por vía oral, las nanopartículas presentan un gran potencial para desarrollar interacciones bioadhesivas con el substrato biológico. Esto permite aumentar el tiempo de residencia de la forma farmacéutica en contacto con la mucosa y facilitar la absorción o la acción de la molécula biológicamente 
activa al (i) aumentar el gradiente de concentración y (ii) minimizar las posibles pérdidas.

Recientemente, la misma unidad ha patentado un procedimiento sencillo para la obtención de nanopartículas del copolímero de metil vinil éter y anhídrido maleico por desolvatación controlada ${ }^{39}$. Este polímero comercializado por ISP bajo la denominación de Gantrez® AN tiene una baja toxicidad y es ampliamente utilizado por la Industria Farmacéutica y Cosmética como agente de recubrimiento de formas sólidas y espesante. Las nanopartículas de este polímero permiten la encapsulación de proteínas. Además, estas nanopartículas se pueden utilizar para obtener conjugados mediante la fijación rápida y eficaz (sin afectar a su actividad) de moléculas o ligandos (por ejemplo lectinas o derivados de la vitamina B12) a la superficie de las mismas. Esta reacción que se realiza por incubación en un medio acuoso, evitando el uso de disolventes o reactivos tóxicos, está basada en la ruptura del grupo anhídrido y en la formación de un enlace éster o amida con un grupo-OH o amino del ligando.

\section{NUEVAS FORMAS FARMACÉUTICAS PARA INMUNOTERAPIA CON ALÉRGENOS}

Para estudiar el perfil inmunológico así como la protección de esta inmunoterapia diseñamos un modelo animal que nos permitiera analizar la respuesta inmune así como la protección de la inmunoterapia con nanopartículas.

Para ello inyectamos por vía intraabdominal dos dosis de $50 \mathrm{mg}$ de alérgeno (ovoalbúmina o Lollium) separadas por 7 días. Una vez sensibilizados los animales, estos se dividieron en cinco grupos que recibieron la inmunoterapia en los días 20, 23 y 26. Como tratamiento de inmunoterapia se utilizaron la siguientes formulaciones: (i) alérgeno encapsulado en nanopartículas de Gantrez®; (ii) alérgeno encapsulado en nanopartículas de Gantrez ${ }^{\circledR}$ conteniendo al que añadimos lipopolisacárido de Brucella ovis; (iii) nanopartículas vacias; (iv) alérgeno en sales de aluminio; y (v) PBS. En el día 41 se realizó una provocación con $4 \mathrm{mg}$ de extracto del alérgeno correspondiente también por vía intraperitoneal. El esquema del modelo animal se representa en la figura 3 .

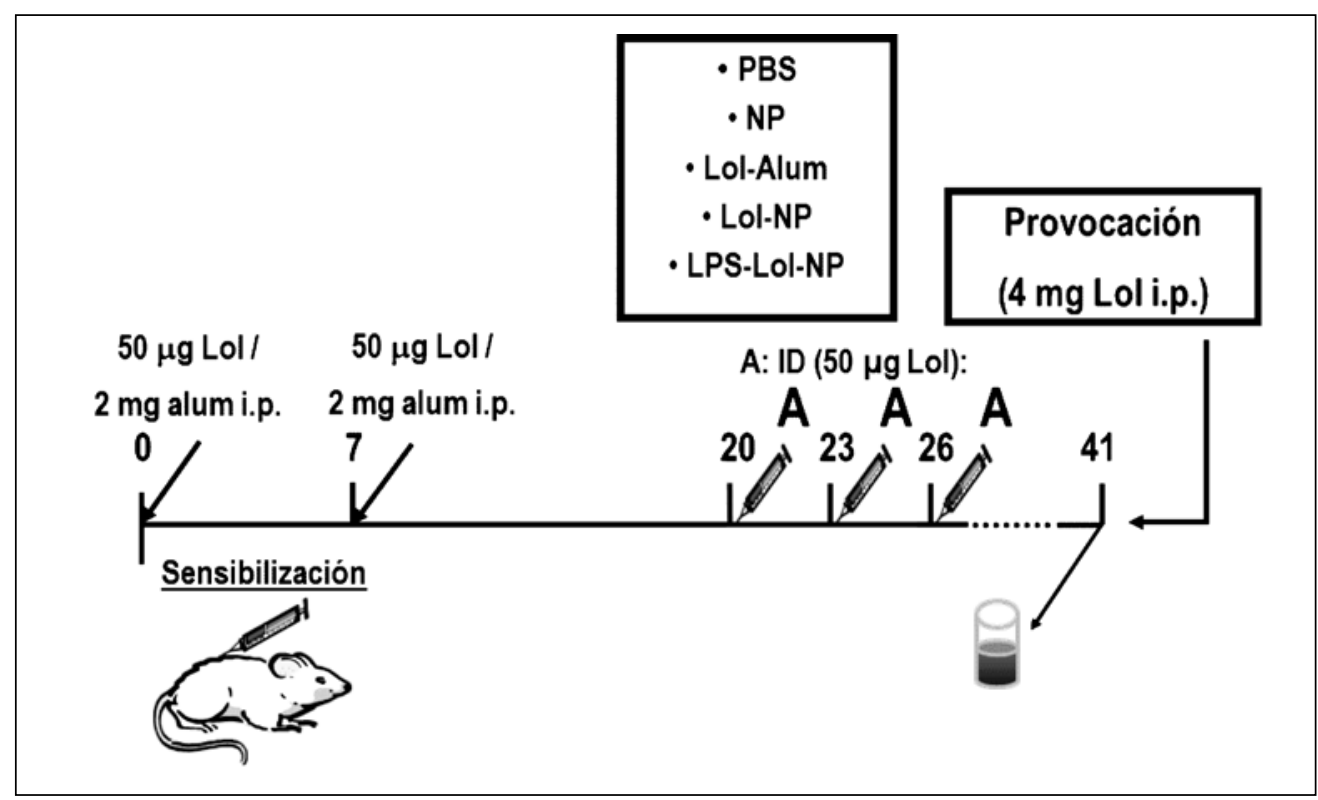

Figura 3. Esquema del modelo animal aplicado. 
Utilizando ovoalbúmina como alérgeno modelo, se pudo observar que cuando las formulaciones de nanopartículas se administraban por vía intradérmica se incrementaba significativamente la respuesta inmunitaria (tanto Th1 como Th2) en ratón $\mathrm{BALB} / \mathrm{c}$ con respecto al control de sales de aluminio ${ }^{21}$. Igualmente, la presencia de lipopolisacárido en las nanopartículas ampliaba significativamente la respuesta Th $1^{42}$. Por otra parte se pudo constatar como las formulaciones basadas en nanopartículas de Gantrez ${ }^{\circledR}$ inducían un aumento significativo de la concentración plasmática de IL-10, citoquina reguladora (Fig. 4).

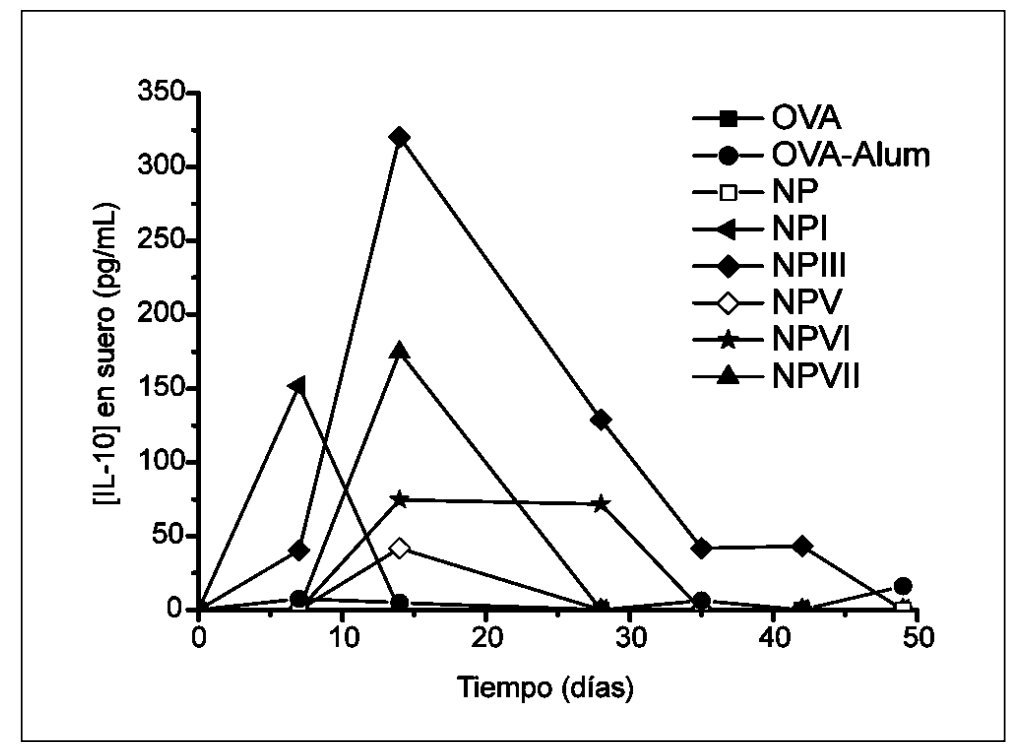

Figura 4. Concentración de IL-10 en suero tras la inmunización de ratones BALB/c por vía intradérmica con las siguientes formulaciones: solución de ovoalbúmina (OVA), ovoalbúmina adsorbida en alhidrogel (OVA-Alum), nanopartículas vacías (NP), NP I, NP III, NP V, NP VI y NP VII.

En otro estudio reciente se analizó el efecto protector frente a anafilaxia que se puede obtener al combinar micropartículas biodegradables, conteniendo ovoalbúmina como alérgeno modelo, y secuencias $\mathrm{CpG}^{40,41}$. Las micropartículas permitieron la co-encapsulación efectiva del alergeno y la secuencia CpG seleccionada. Tras su administración por vía intradérmica, se pudo constatar cómo esta estrategia era adecuada para proteger frente a un desafío tras la administración intraperitoneal del alergeno modelo.

Una vez comprobada la protección por vía tradicional, se exploró la eficacia de estos adyuvantes particulados tras su admi- nistración por vía oral. Se pudo comprobar como ciertas formulaciones de nanopartículas se revelaron como las más eficaces a la hora de inducir una protección frente a la muerte por shock anafiláctico inducido por ovoalbúmina ${ }^{42}$.

Finalmente, con los resultados obtenidos, se evaluó la capacidad de dichas nanopartículas como adyuvantes mediante la utilización de un alérgeno inhalante. Se pudo comprobar como la encapsulación de los alérgenos mayores del polen de Lollium perenne (responsable de la alergia primaveral) en nanopartículas de Gantrez ${ }^{\circledR}$, daba lugar a vehículos con características físico-químicas y estabilidad similares a las 
nanopartículas obtenidas con ovoalbúmi$\mathrm{na}^{43}$. Se probaron dos tipos de nanopartículas conteniendo los alérgenos de Lollium perenne, con y sin lipopolisacárido de Brucella ovis como inmunomodulador. Dichas formulaciones se ensayaron en el modelo animal que previamente se había sensibilizado a este polen. Se pudo observar como las nanopartículas conteniendo LPS eran capaces de inducir una respuesta Th1 más potente (Fig. 5). Además, por vía intradérmica, las formulaciones protegieron frente a un desafío con este alérgeno en el mode- lo de animal alérgico. Así, se pudo constatar como los animales inmunizados con la formulación de nanopartículas mostraban signos de anafilaxia (descenso de temperatura, movilidad) mucho menos contundentes que los animales control. Igualmente los niveles plasmáticos de la proteasa de mastocitos murinos 1 (mMCP-1), enzima asociada a la existencia de un fuerte shock anafiláctico, eran significativamente menores para los animales tratados con la formulación de nanopartículas que los de los animales control (Fig. 6).

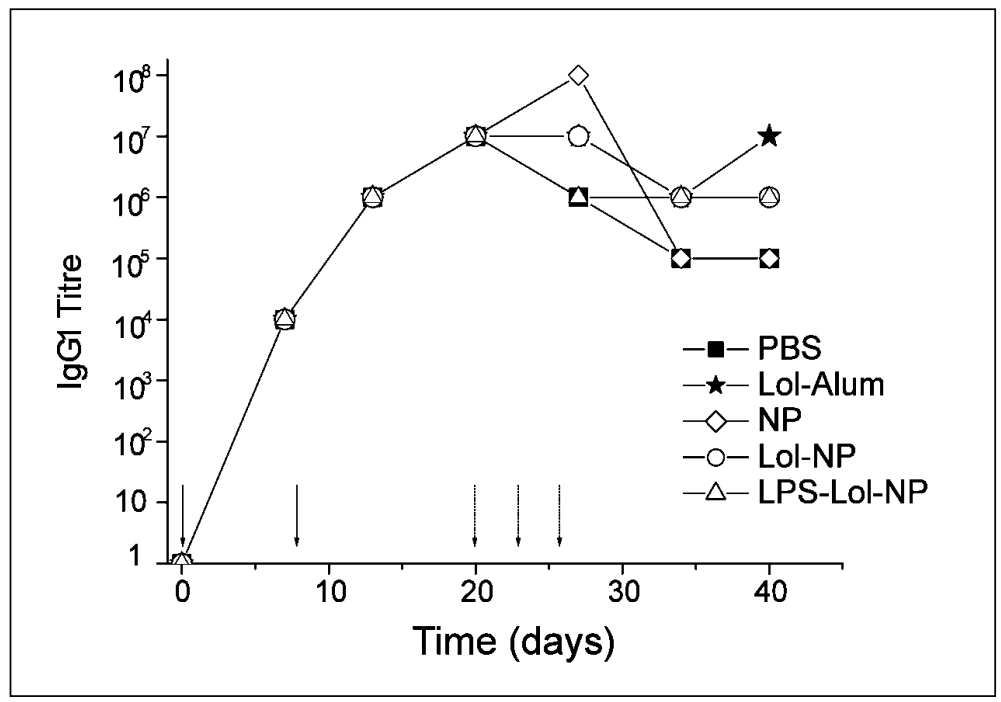

Figura 5. Inmunogenicidad de las fórmulas empleadas. Una vez los ratones fueron sensiblizados con dos inyecciones intraperitoneales de extracto de Lollium. Se dividieron en cinco grupos que recibieron los días 20, 23 y 26 Lollium encapsulado en nanopartículas Gantrez® (Lol-NP), Lollium encapsulado al que añadimos lipopolisacárido de Brucella ovis (LPS-Lol-NP), nanopartículas sin alérgeno (NP), Lollium en aluminio (Lol-Alum) y PBS respectivamente extrayéndose muestra sanguínea en esos días y analizándose el perfil de anticuerpos, IgG1 el cual reflejaba un perfil Th2 e IgG2 correspondiente a perfil Th1. 

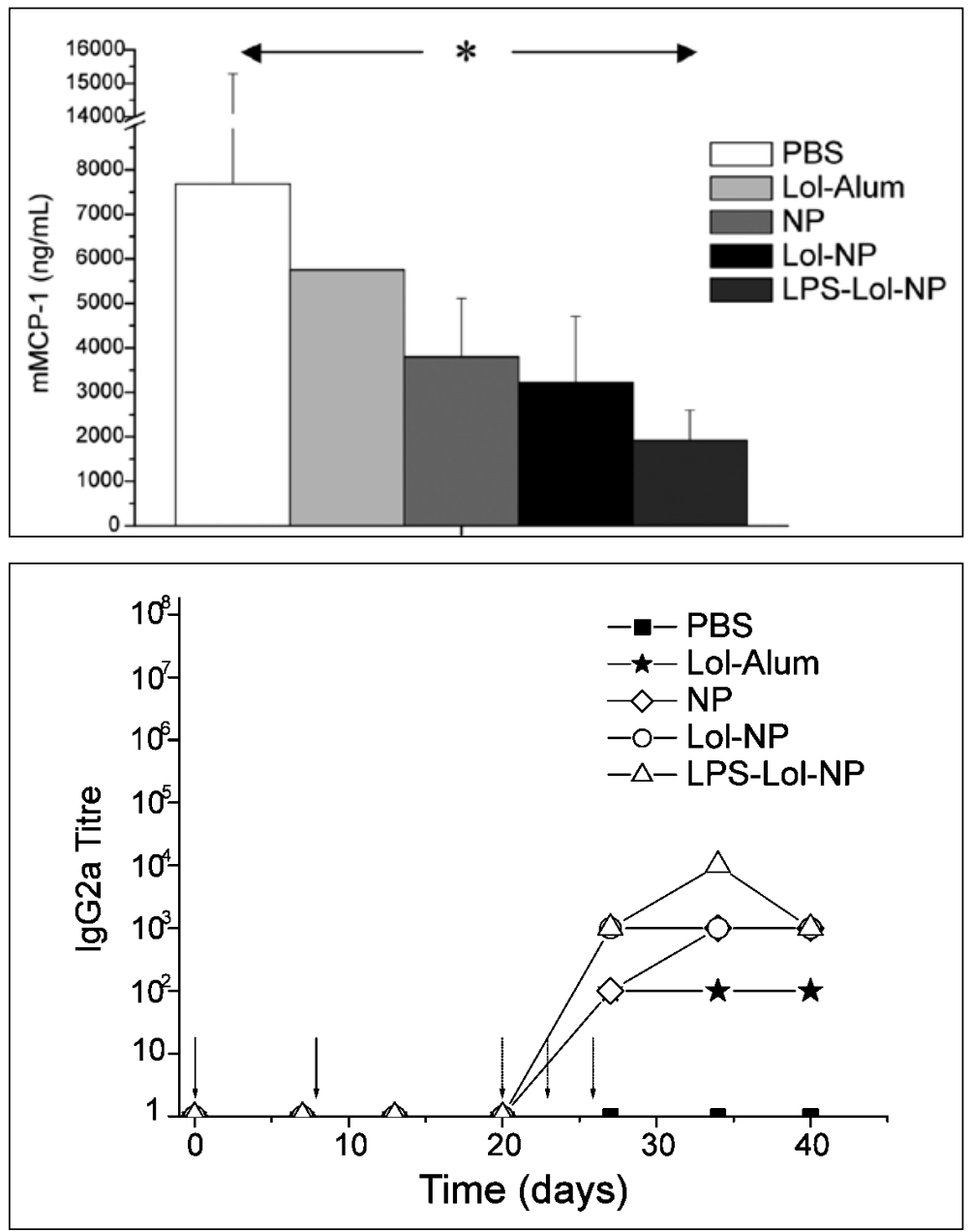

Figura 6. Niveles de mMCP-1 tras provocación con Lollium perenne. Observamos diferencias significativas al ensayar el alérgeno encapsulado con nanopartículas más lipopolisacárido de Brucella ovis.

\section{APLICACIÓN PARA EL}

\section{TRATAMIENTO DE ALERGIA}

\section{ALIMENTARIA}

Además del tratamiento de la alergia causada por agentes inhalantes, las micro y nanopartículas tienen un gran potencial en el diseño de inmunoterapia por vía oral. La alergia alimentaria se ha convertido en un problema de salud de gran importancia ${ }^{44}$. El impacto social de esta patología ha hecho que este tema se haya incluido en el VII programa Marco de la UE.
El único medio disponible en el momento actual para tratar la hipersensibilidad a alimentos es la restricción estricta del contacto con el alimento implicado. La dificultad y la alteración de la calidad de vida viene dada porque cantidades mínimas o inapreciables desencadenan esta reacción inmunológica por lo que la vigilancia debe ser permanente y extrema. Una vez detectado el alimento implicado, la mayoría de reacciones anafilácticas se deben a ingestas accidentales ${ }^{45}$. 
En alergia alimentaria no existía la posibilidad al administrar una inmunoterapia hasta hace pocos años; desde entonces se ha hecho un gran esfuerzo en investigación para desarrollo. Esta inmunoterapia consiste en la inducción de tolerancia mediante pautas de desensibilización ${ }^{46}$. Estas pautas consiguen, a través de la administración de dosis crecientes del alimento implicado, que el paciente tolere mínimas cantidades del mismo. En la última revisión sistemática de seis estudios publicada se concluye que para muchos pacientes esta inmunoterapia les permite incrementar el umbral de tolerancia durante el tratamiento, si bien las reacciones adversas fueron frecuentes durante la inmunoterapia, siendo la mayoría leves aunque algunas con riesgo vital. En conclusión, aunque parece ofrecer resultados prometedores, se recomienda realizar más estudios para demostrar la eficacia y coste-beneficio especialmente a largo plazo, debiéndose realizar estudios más potentes que permitan recomendar su uso clínico ${ }^{47}$.

Por las limitaciones que hemos mencionado, se están explorando nuevas formas de administrar la inmunoterapia de forma que mejore su seguridad y eficacia. En este sentido ha cobrado protagonismo el uso de nuevas vías de administración y en este momento para alérgenos por vía inhalatoria se cuenta con inmunoterapia sublingual y en comprimidos.

\section{CONSIDERACIONES FINALES}

En definitiva, las nuevas formas farmacéuticas -nanopartículas, micropartículas, liposomas- son vehículos eficaces para la encapsulación de alérgenos, inducen una respuesta Th1, y ofrecen protección frente a la provocación con alérgeno en modelos animales. Permiten asimismo incorporar coadyuvantes como el lipopolisacárido de Brucella ovis (LPS-R) o secuencias CpG que hacen que ese efecto buscado sea todavía más potente. Por lo tanto, pueden jugar un papel prometedor en el tratamiento de las enfermedades alérgicas, y, potencialmente en enfermedades autoinmunes.

\section{BIBLIOGRAFÍA}

1. KUSCHNER WG. The asthma epidemic. N Engl J Med 2007; 356: 1073.

2. Asher Mi, Montefort S, BJorksten B, Lai CK, Strachan DP, Weiland SK et al. Worldwide time trends in the prevalence of symptoms of asthma, allergic rhinoconjunctivitis, and eczema in childhood: ISAAC Phases One and Three repeat multicountry cross-sectional surveys. Lancet 2006; 368: 733-743.

3. Fernandez Rivas M. Food allergy in Alergologica-2005. J Investig Allergol Clin Immunol 2009; 19 (Suppl. 2): 37-44.

4. Navarro A, Colas C, Anton E, Conde J, Davila I, DORDAL MT et al. Epidemiology of allergic rhinitis in allergy consultations in Spain: Alergologica-2005. J Investig Allergol Clin Immunol 2009; 19 (Suppl. 2): 7-13.

5. Radulovic SCM, Wilson D, Durham S. Sublingual immunotherapy for allergic rhinitis. Cochrane Database Syst Rev 2010; CD002893.

6. CaLderon MA AB, Jacobson M, HuRwitz B, SheiKH A, Durham S. Allergen injection immunotherapy for seasonal allergic rhinitis. Cochrane Database Syst Rev 2007; CD001936.

7. TsitouRA DC, TAssios Y. Immunomodulation: the future cure for allergic diseases. Ann N Y Acad Sci 2006; 1088: 100-115.

8. FonseCA DE KJ. Use of CpG oligonucleotides in treatment of asthma and allergic disease. Adv Drug Deliv Rev. 2009; 61: 256-262.

9. SheiKh NA, al-Shamisi M, Morrow WJ. Delivery systems for molecular vaccination. Curr Opin Mol Ther 2000; 2: 37-54.

10. Clements CJ, Griffiths E. The global impact of vaccines containing aluminium adjuvants. Vaccine 2002; 20 (Suppl. 3): S24-S33.

11. Francis MJ, Fry CM, Rowlands DJ, Bittle JL, HOUGHTEN RA, LERNER RA et al. Immune response to uncoupled peptides of foot-and-mouth disease virus. Immunology 1987; 61: 1-6.

12. Kwissa M, Lindblad EB, Schirmbeck R, Reimann J. Codelivery of a DNA vaccine and a protein vaccine with aluminum phosphate stimulates a potent and multivalent immune response. J Mol Med (Berl) 2003; 81: 502-510.

13. Schisns V E. Immunological concepts of vaccine adjuvant activity. Curr Opin Immunol 2000; 12: 456-63.

14. O'Hagan DT, Valiante NM. Recent advances in the discovery and delivery of vaccine adjuvants. Nat Rev Drug Discov 2003; 2: 727-735.

15. Kersten G, Hirschberg H. Antigen delivery systems. Expert Rev Vaccines 2004; 3: 453-462. 
16. ULMER JB. Enhancement of vaccine potency through improved delivery. Expert Opin Biol Ther 2004; 4: 1045-1051.

17. Calderon MA, Penagos M, Sheikh A, Canonica GW, Durham S. Sublingual immunotherapy for treating allergic conjunctivitis. Cochrane Database Syst Rev 2011; CD007685.

18. Radulovic S, Wilson D, Calderon M, Durham S. Systematic reviews of sublingual immunotherapy (SLIT). Allergy 2011; 66: 740-752.

19. Enrique E, Pineda F, Malek T, Bartra J, Basagana M, Tella R et al. Sublingual immunotherapy for hazelnut food allergy: a randomized, double-blind, placebo-controlled study with a standardized hazelnut extract. J Allergy Clin Immunol 2005; 116: 1073-1079.

20. Fernández-Rivas M, Garrido Fernandez S, Nadal JA, Diaz de Durana MD, García BE, GonzalezMancebo E et al. Randomized double-blind, placebo-controlled trial of sublingual immunotherapy with a Pru p 3 quantified peach extract. Allergy 2009; 64: 876-883.

21. Gómez S, Gamazo C, San Román B, Vauthier C, FERRER M, IRACHE JM. Developement of a novel vaccine delivery system based on Gantrez nanoparticles. J Nanosci Nanotechnol 2006; 6: 3283-3289.

22. Rice-Ficht AC, AREnas-Gamboa AM, Kahl-McDonagh MM, Ficht TA. Polymeric particles in vaccine delivery. Curr Opin Microbiol 2010; 13: 106-112.

23. Chadwick S, Kriegel C, AmiJi M. Nanotechnology solutions for mucosal immunization. Adv Drug Deliv Rev 2010; 62: 394-407.

24. ZolniK BS, Gonzalez-Fernandez A, Sadrieh N, DoBROVOLSKAIA MA. Nanoparticles and the immune system. Endocrinology 2010; 151: 458-465.

25. WILSON KD, DE JONG SD, TAM YK. Lipid-based delivery of $\mathrm{CpG}$ oligonucleotides enhances immunotherapeutic efficacy. Adv Drug Deliv Rev 2009; 61: 233-242.

26. Altin JG, PARISH CR. Liposomal vaccines-targeting the delivery of antigen. Methods 2006; 40: 39-52.

27. Dow S. Liposome-nucleic acid immunotherapeutics. Expert Opin Drug Deliv 2008; 5: 1124.

28. Tovey MG, Lallemand C. Adjuvant activity of cytokines. Methods Mol Biol 2010; 626: 287309.

29. Gamvrellis A, Leong D, Hanley JC, Xiang SD, Mottram P, Plebanski M. Vaccines that facilitate antigen entry into dendritic cells. Immunol Cell Biol 2004; 82: 506-516.
30. Singh M, Chakrapani A, O'Hagan D. Nanoparticles and microparticles as vaccine-delivery systems. Expert Rev Vaccines 2007; 6: 797808.

31. Scholl I, Weissenbock A, Forster-Waldl E, Untersmayr E, Walter F, Willheim M et al. Allergenloaded biodegradable poly (D,L-lactic-co-glycolic) acid nanoparticles down-regulate an ongoing Th2 response in the BALB/c mouse model. Clin Exp Allergy 2004; 34: 315-321.

32. Batanero E, Barral P, Villalba M, Rodríguez R. Encapsulation of Ole e 1 in biodegradable microparticles induces Th1 response in mice: a potential vaccine for allergy. J Control Release 2003; 92: 395-398.

33. Ropert C, Malvy C, Couvreur P. Inhibition of the Friend retrovirus by antisense oligonucleotides encapsulated in liposomes: mechanism of action. Pharm Res 1993; 10: 1427-1433.

34. O'HAGAN DT, RAPPUOLI R. Novel approaches to vaccine delivery. Pharm Res 2004; 21: 15191530.

35. Singh M, O'Hagan D T. Recent advances in vaccine adjuvants. Pharm Res 2002; 19: 715-728.

36. Alvarez MJ, Echechipia S, Garcia B, Tabar AI, Martin S, Rico P et al. Liposome-entrapped D. pteronyssinus vaccination in mild asthma patients: effect of 1-year double-blind, placebo-controlled trial on inflammation, bronchial hyperresponsiveness and immediate and late bronchial responses to the allergen. Clin Exp Allergy 2002; 32: 1574-1582.

37. Basomba A, Tabar AI, de Rojas DH, Garcia BE, Alamar R, Olaguibel J M et al. Allergen vaccination with a liposome-encapsulated extract of Dermatophagoides pteronyssinus: a randomized, double-blind, placebo-controlled trial in asthmatic patients. J Allergy Clin Immunol 2002; 109: 943-948.

38. Spenlehauer G, Spenlehauer-Bonthonneau F, Thies C. Biodegradable microparticles for delivery of polypeptides and proteins. Prog Clin Biol Res 1989; 292: 283-291.

39. Arbos P, Wirth M, Arangoa M A, Gabor F, IraCHE J M. Gantrez AN as a new polymer for the preparation of ligand-nanoparticle conjugates. J Control Release 2002; 83: 321-330.

40. San Roman B, Irache JM, Gomez S, Gamazo C, EsPUElas S. Co-Delivery of Ovalbumin and $\mathrm{CpG}$ motifs into microparticles protected sensitized mice from anaphylaxis. Int Arch Allergy Immuno 2009; 149: 111-118.

41. San Roman B, Irache JM, Gomez S, Tsapis N, GaMAZO C, Espuelas MS. Co-encapsulation of an antigen and $\mathrm{CpG}$ oligonucleotides into PLGA 
microparticles by TROMS technology. Eur J Pharm Biopharm 2008; 70: 98-108.

42. Gómez S, Gamazo C, Roman BS, Ferrer M, Sanz ML, IRACHE JM. Gantrez AN nanoparticles as an adjuvant for oral immunotherapy with allergens. Vaccine 2007; 25: 5263-5271.

43. Gómez S, Gamazo C, San Roman B, Grau A, EspueLAS S, Ferrer M et Al. A novel nanoparticulate adjuvant for immunotherapy with Lolium perenne. J Immunol Methods 2009; 348: 1-8.

44. LAck G. Clinical practice. Food allergy. N Engl J Med 2008; 359: 1252-1260.
45. De Silva IL, Mehr SS, Tey D, Tang ML. Paediatric anaphylaxis: a 5 year retrospective review. Allergy 2008; 63: 1071-1076.

46. Clark AT, Islam S, King Y, Deighton J, Anagnostou K, EWAN PW. Successful oral tolerance induction in severe peanut allergy. Allergy 2009; 64: 1218-1220.

47. Sheikh A, Nurmatov U, Venderbosch I, Bischoff E. Oral immunotherapy for the treatment of peanut allergy: systematic review of six case series studies. Prim Care Respir J 2011; 21: 41-49 
